\title{
How does vessel curvature influence drug release from DES and drug transport in arterial tissue?
}

\author{
Javier Escuer ${ }^{1}$, Irene Aznar ${ }^{1}$, Estefanía Peña ${ }^{1,2}$, Miguel Ángel Martínez ${ }^{1,2}$ \\ ${ }^{1}$ Applied Mechanics and Bioengineering \\ Aragón Institute of Engineering Research (I3A) \\ University of Zaragoza, Mariano Esquillor s/n, 50018, Zaragoza, Spain. \\ Tel. +34-976762707, e-mail: jescuer@unizar.es \\ ${ }^{2}$ Biomedical Research Networking Center in Bioengineering, Biomaterials and Nanomedicine (CIBER-BBN)
}

\begin{abstract}
Several computational models of transport of drugs eluted from drug-eluting stents (DES) in curved arteries were developed in order to investigate the influence of the arterial curvature and complex geometries on drug transport in the blood flow and in the arterial wall.
\end{abstract}

\section{Introduction}

Coronary heart disease (CHD) is the leading cause of death in the world. CHD is attributed to atherosclerosis, which is characterized by the accumulation of lipids and fibrous elements inside the arteries causing a progressive reduction in the lumen available for blood flow (stenosis). Stent implantation has become the most successful strategy in the treatment of advanced atherosclerotic lesions in arteries. However, in-stent restenosis (ISR), a gradual luminal re-narrowing mainly due to the vessel wall injury induced by the device, is the major clinical limitation of this technique. With the advent of drug-eluting stents (DES), the incidence of restenosis has been dramatically reduced to less than $10 \%$. However, DES do not completely remove this problem. Several computational studies have been performed in the last decade to investigate drug-release and binding mechanisms in arteries, but they usually represent simplified straight vessel geometries. However, actual arteries, and specially coronary arteries, are highly curved vessels. Therefore, the main aim of this contribution is to analyse the influence of curvature and complex geometries on drug transport in the arterial wall.

\section{Materials and Methods}

We develop several 2D computational models of coupled stent drug release and redistribution in the arterial wall, similar to that employed by Bozsak et al. [1] but with important differences: we consider the adventitia as a layer of the model instead of as a boundary condition, a curved geometry of the vessel and a non-linear saturable reversible reaction model in the tissue to describe drug specific binding to receptors and drug non-specific binding to general extracellular matrix sites $[2,3]$. The computational geometry consists of an idealized curved segment of a coronary artery with ten circular struts embedded in the tissue. In the lumen, blood is treated as a Newtonian fluid and blood flow is assumed to be steady, laminar and incompressible and is modelled by steady Navier-Stokes equations. The arterial wall is modelled as a multilayer anisotropic porous structure distinguishing three different domains: subendothelial space (SES), media and adventitia. Darcy's law is used to calculate plasma filtration through the porous layers and convection-diffusionreaction equations are used to model drug transport through blood and the distinct layers of the tissue. Endothelium and internal and external elastic laminae are considered as semipermeable membranes and the flux across them is described by Kedem-Katchalsky equations. The majority of DES to date have contained either the drug paclitaxel, sirolimus or a sirolimus analogue. In this work we consider both sirolimus and paclitaxel. The effect of the stent struts embedment configuration and stent malapposition is also studied in this work. Wherever possible, model input parameters were derived from experimental data available in the literature. The commercial software package COMSOL Multiphysics 5.3 (COMSOL AB, Burlington, MA, USA) was used to create the computational geometry and to solve numerically the partial differential equations by means of the finite element method (FEM).

\section{Results}

As example of the obtained results the time-varying normalized mean concentration (NMC) profiles of paclitaxel in the media layer of the arterial wall for the different curvatures studied are shown in Fig. 1. These profiles allow observing the different 
concentration existing in the internal and external sides of the artery.

\section{Conclusions}

The arterial curvature affects the drug concentration in the tissue few hours after stent implantation, increasing the levels of drug in the internal side of the vessel and decreasing in the external one. The internal concentration can be increased until two times the external one and this ratio is magnified for higher curvatures. The stent malaposition, usually associated to tortuous vessels, also modified the levels of drug in the arterial wall and can affect the efficacy of the treatment.

\section{Acknowledgments}

The authors would like to gratefully acknowledge the funding provided by the Spanish Ministry of
Economy, Industry and Competitiveness under research project number DPI2016-76630-C2-1-R and the Instituto de Salud Carlos III (ISCIII) through the CIBER initiative.

\section{REFERENCES}

[1]. BOZSAK, F., CHOMAZ, J.M., BARAKAT, A.I. Modeling the transport of drugs eluted from stents: physical phenomena driving drug distribution in the arterial wall. Biomechanics and Modelling in Mechanobiology. 2014, 13(2), 327-347.

[2]. McGINTY, S., PONTRELLI, G. A general model of coupled drug release and tissue absortion for drug delivery devices. Journal of Controlled Release. 2015, 217:327-336.

[3]. TZAFrIRI, A.R, GROOTHUIS, A., PRICE, G.S., EDELMAN, E.R. Stent elution rate determines drug deposition and receptor-mediated effects. Journal of Controlled Release. 2012, 161(3):918-926.

\section{FIGURES}
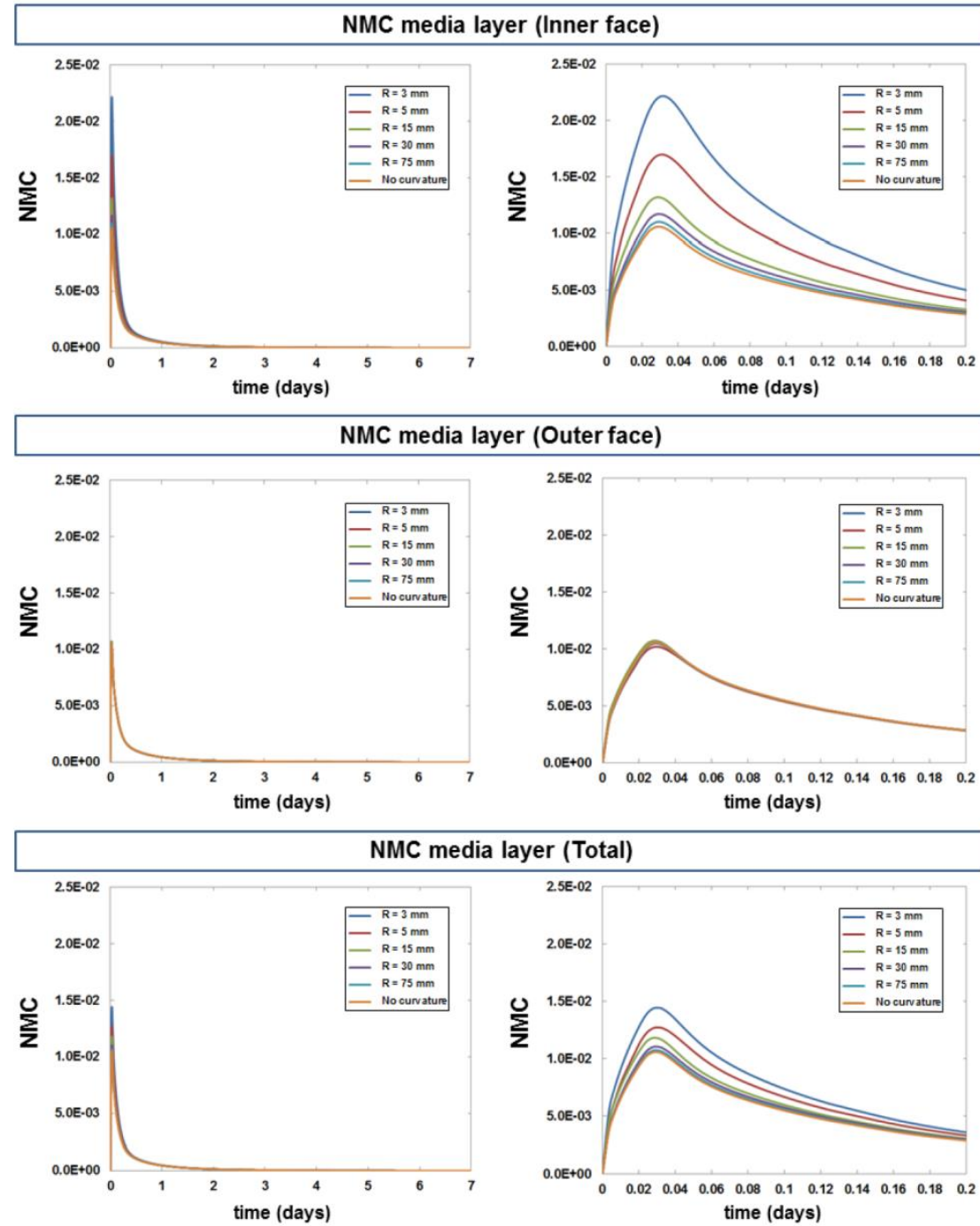

Figure 1. Time-varying normalized mean concentration (NMC) profiles of paclitaxel in the media layer of the arterial wall for the different curvatures studied in this work.

Revista "Jornada de Jóvenes Investigadores del I3A", vol. 7 (Actas de la VIII Jornada de Jóvenes Investigadores del I3A - 6 de junio de 2019). ISSN 2341-4790. 